\title{
Tangence
}

\section{L'amour moderne : de tradition en transgression ou... la féminité en question}

\section{Pascale Noizet}

Numéro 47, mars 1995

Écritures au féminin : le genre marqué

URI : https://id.erudit.org/iderudit/025847ar

DOI : https://doi.org/10.7202/025847ar

Aller au sommaire du numéro

Éditeur(s)

Tangence

ISSN

0226-9554 (imprimé)

1710-0305 (numérique)

Découvrir la revue

Citer cet article

Noizet, P. (1995). L'amour moderne : de tradition en transgression ou... la

féminité en question. Tangence, (47), 8-20. https://doi.org/10.7202/025847ar d'utilisation que vous pouvez consulter en ligne.

https://apropos.erudit.org/fr/usagers/politique-dutilisation/ 


\section{L'amour moderne: de tradition en transgression ou... la féminité en question Pascale Noizet, Université d'Ottawa}

En 1970, dans un essai intitulé La dialectique du sexe, Shulamith Firestone affirme: "Les femmes et l'amour sont la pierre angulaire de la civilisation. Les soumettre à examen, c'est menacer la structure même de la civilisation." L'assertion est exacte et elle est également lourde de conséquences de par la dimension épistémologique qu'elle soutient. Il s'agit en effet de reconnaître l'amour, non pas comme un donné naturel, mais bien comme un construit dont le principe repose, depuis le XVII ${ }^{e}$ siècle, sur une organisation fortement structurée des rapports sociaux de sexe ${ }^{2}$. Au niveau littéraire, on peut reconnaître un nouveau type de récit qui émerge à l'aune de l'idée moderne d'amour. Il est possible, en celte fin de siècle, d'en appréhender la norme générique à même le corpus Harlequin. Ici, une précision s'impose. La tentation est forte de considérer les romans Harlequin et Cie comme les seuls dépositaires de la thématique sentimentale. Rien n'est plus faux et nous ne donnerons qu'un seul exemple pour montrer que l'hégémonie de l'idée moderne d'amour se fonde sur une division du travail discursif.

Il s'agit donc d'un cas exemplaire, celui du film intitulé $L a$ leçon de piano réalisé par Jane Campion. Ce film a reçu l'hommage d'une critique qui, en grande majorité, a en loué l'excellence en faisant fi des questions fondamentales que soulevait son scénario. Or, structuralement parlant, La leçon de piano nous expose un scénario qui s'identifie à celui des romans Harlequin. En effet, le sentiment évolue au sein d'une relation de pouvoir, à savoir un chantage sexuel exercé précisément sur la protagoniste principale. L'histoire se termine sur une note idyllique - et là

1 Shulamith Firestone, La dialectique du sexe, traduit de l'anglais par Sylvia Gleadow, Paris, Stock, 1970, p. 161.

2. Pour une analyse plus approfondie de l'idée moderne d'amour, nous renvoyons à notre thèse de doctorat: Pascale Noizet, L'idée modeme d'amour. Entre sexe et genre: vers une théorie du sexologème, Montréal, Université du Québec à Montréal, 1993, 433 p. 
réside le scandale idéologique de l'amour moderne - qui fera l'impasse sur la reconnaissance de ce rapport de pouvoir: la protagoniste aime son... agresseur sexuel! Le mince filet des voix dissidentes qui se sont fait entendre a été promptement noyé par la " censure universelle" :

[...] dans chaque société, l'interaction des discours, les intérêts qui les soutiennent et la nécessité de * penser • collectivement la nouveauté historique finissent par produire une hégémonie sans cesse en voie de réfection, qui surdétermine globalement une grande partie de ce qui est pensable/énonçable et surtout prive de moyens d'énonciation l'impensable, le nocb-nicht gedachtes (pour adapter Bloch) qui ne correspond aucunement cependant avec l'inexistant ou le chimérique. ${ }^{3}$

Certaines voix prennent cependant le risque de traquer effrontément l'énoncé doxal. L'intérêt du présent article porte sur celleslà mêmes qui ont opéré une transgression de l'habitus sentimental. En effectuant d'abord une synthèse de la tradition sentimentale moderne, nous traiterons ici de trois productions romanesques qui bouleversent ou bousculent, chacune de façon distincte, cette tradition. Qu'elles exercent une plume satirique, c'est le cas de Jane Austen ${ }^{4}$, ou parodique comme Louise Leblanc ${ }^{5}$, qu'elles insistent sur sa marque concentrationnaire à la façon d'Elfriede Jelinek ${ }^{6}$, nous verrons en effet comment ces productions, écrites par des femmes, montrent ou démontent, selon le cas, le piège essentialiste de la féminité.

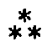

En son origine littéraire, l'amour moderne est directement saisissable à même la formation générique du roman pathéticosentimental dont Paméla ou la vertu récompensée écrit en 1740

3 Marc Angenot, :Le discours social: problématique d'ensemble *, Cabiers de recberche sociologique, vol. II, $\mathrm{n}^{\circ} 1$, avril 1984, p. 32.

4 Jane Austen, Catberine Morland. Northanger Abbey [1818], traduit de l'anglais par Félix Fénéon, Paris, Gallimard, 1946, 253 p.

5 Louise Leblanc, 37 1/2 AA [1983], Montréal, Quinze, coll. • 10/10 •, 1985, 203 p.

6 Elfriede Jelinek, Les amantes [1975], traduit de l'allemand par Yasmin Hoffmann et Maryvonne Litaize, Nimes, Éditions Jacqueline Chambon, 1992, 221 p.

7 Samuel Richardson, Paméla ou la vertu récompensée [1740], traduit de l'anglais par l'abbé Prévost, Paris, Nizet, 1977, 378 p. 
par Samuel Richardson est l'exemple le plus représentatif. Ce qu'il est important de souligner ici, c'est que le genre pathéticosentimental qui émerge au XVIII ${ }^{\mathrm{e}}$ siècle opère un recentrage de l'amour sur les positions de sexe, franchissant la frontière des classes sociales. L'histoire se réalise dorénavant à l'intérieur du couple - elle se privatise en quelque sorte - et cette nouvelle éthique organise un matériau romanesque qui concentre son récit sur une héroïne problématique dont l'amour au sein de la relation hétérosexuelle sera le principal terrain d'expérience. À l'idée d'épreuve qui caractérise l'histoire sentimentale préindustrielle se surajoute l'idée de formation et d'apprentissage. Mais, là réside la nouveauté structurale, l'épreuve autrefois actualisée entre le couple amoureux et l'ordre social prégnant se réalise cette fois par l'antagonisme formateur entre les deux protagonistes: ce rétrécissement du monde s'organise sur un réseau sémiotique propre à la relation hétérosexuelle puisque celle-ci devient constitutive du rapport amoureux. $\grave{A}$ ce niveau, la logique qui ordonne l'intelligibilité de l'amour moderne se fonde sur le procès d'une différenciation ${ }^{8}$ sexuée au sein du couple. Loin de transgresser l'ordre social comme il le faisait dans les annales de la féodalité, le sentiment amoureux s'intègre au contraire à un nouvel état de société. Mais il fait plus que s'intégrer, il définit et supporte en fait de nouvelles normes sexuées et ce, dans une visée didactique tournée vers les femmes.

Dans son principe, le roman d'amour moderne oriente fondamentalement la constitution du rapport amoureux sur l'antagonisme des classes de sexe et le conduit à son obligatoire résolution. Nous passons de l'imposition du mariage de convenance à la finale idyllique du mariage que l'on dit d'amour et qui sousentend l'exercice d'un libre arbitre. Entre la rencontre initiale et l'union finale, la prégnance de la confrontation informe l'antagonisme du rapport hétérosexuel et le construit. Pour résoudre ce rapport contradictoire, le récit recourt à un mode particulier d'énonciation narrative dont l'exécution se fonde sur le principe d'une partialité catégorielle, en l'occurrence la focalisation interne centrẹe sur l'héroïne. Cette focalisation aura la fonction idéolo-

8 La différenciation, c'est: $\cdot[. .$.$] assigner aux deux sexes des fonctions diffé-$ rentes dans le corps social. . Voir Nicole-Claude Mathieu, .Différenciation des sexes., Dictionnaire de l'ethnologie et de l'anthropologie, Paris, PUF, 1991, p. 660-664. 
gique de lui faire supporter l'histoire sentimentale. Cette modélisation est un élément essentiel de compréhension de l'amour moderne. Ce type narratif actoriel à dominante féminine rentabilise la division sexuelle de l'amour puisqu'il s'oriente en l'exacerbant sur l'identité de la protagoniste principale: tout se passe comme si l'amour l'enfermait dans une nature spécifique dont la force d'intoxication l'empêche d'évaluer sa situation, cette nature prenant le dessus sur la conscience de l'oppression. En fait, en passant quasiment du sacré au profane, l'amour devient au xvIII ${ }^{\mathrm{e}}$ siècle un fondement structural de la construction hétérosociale? des catégories de sexe, et dans ce sens il fait en sorte de devenir un élément constitutif de la féminité.

Ainsi, à partir du xviI ${ }^{e}$ siècle, le récit sentimental moderne s'applique à différencier les sexes tout en emprisonnant, de façon spécifique, le personnage féminin dans l'amour. On connaît bien, à l'heure actuelle, la force idéologique des éditions Harlequin dont les 220 millions d'exemplaires émigrent annuellement dans plus de 90 pays $^{10}$ : c'est un lectorat essentiellement féminin qui subit les effets de cette multinationale du cœur. Dans ce sens, Harlequin est l'exemple représentatif d'un amour occidental qui fonctionne bien comme un principe de catégorisation des sexes. À ce niveau même, rares sont les récits sentimentaux qui transgressent la norme élaborée par le système de sexe/genre ${ }^{11}$. Il en est cependant qui ont compris l'importance de leur remise en question, ainsi que nous allons le voir dans les pages qui suivent.

9 Nous définissons l'hétérosocialité comme étant l'organisation dialectique des deux catégories de sexe dont le procès de différenciation fonde la définition des femmes et de leur oppression. Nous nous inspirons ici dès théories développées par Monique Wittig (.La pensée straight•, Questions féministes, $\mathrm{n}^{\circ} 7$, p. 45-53; . On ne naît pas femme - Questions féministes, n 8, p. 75-84; - The Category of Sex •, Feminist Issues, vol. II, $n^{\circ} 2$, p. 63-68) et celles de Nicole-Claude Mathieu ("Identité sexuelle/sexuée/de sexe? Trois modes de conceptualisation du rapport entre sexe et genre., Catégorisation de sexe et constructions scientifiques, Aix-en-Provence, CEFUP, 1989, p. 109-146).

10 Ces chiffres proviennent de la maison d'édition canadienne.

11 L'expression de sex/gender system a été créée par Gayle Rubin. Pour elle, le sex/gender system agit comme un tabou contre la similitude des hommes et des femmes, en divisant les sexes en deux catégories exclusives. Gayle Rubin, - The Traffic in Women: Notes on the "Political Economy" of Sex *, Toward an Anthropology of Women, New York, London, Monthly Review Press, 1975, P. 157-210. 


\section{***}

Jane Austen, dont on reconnaît habituellement la verve satirique, s'est intéressée de très près aux sentiments amoureux. Dans la mouvance d'un $\mathrm{xIX}^{\mathrm{e}}$ siècle qui s'annonce fort romantique, sa plume acerbe repère avec efficacité les raisons économiques de ces nouvelles amours et la destinée obligée de ses héroïnes. Catberine Morland. Northanger Abbey est un roman qu'elle peaufine durant dix-sept ans (de 1798 à 1815). Édité en 1818, cette œuvre posthume raconte l'histoire de Catherine Morland: *Personne qui ait jamais vu Catherine Morland dans son enfance ne l'aurait supposée née pour être une héroïne" 12 . Dans ses débuts, le récit insiste sur le caractère ordinaire de l'héroïne, interpellant par le fait même le portrait sémantique de la protagoniste des romans d'amour: portrait physique et intellectuel très moyen qui ne convoque aucun destin d'envergure. Catherine Morland vient rejoindre la plupart de ses consœurs qu'il faut banaliser pour mieux les incarcérer dans le micromonde de la vie privée. Ici, la distanciation satirique sert une critique qui n'est pas de bon aloi envers la tradition littéraire et surtout envers le sort qu'elle réserve à ses personnages féminins. Visionnaire, le texte de Jane Austen rejoint le constat que feront, quelque deux cents ans plus tard, les analyses féministes ${ }^{13}$. Il sous-entend en fait que les héroïnes n'ont rien d'hérö̈que!

Cependant, le portrait sémantique initial de Catherine Morland ne suit pas des aléas conventionnels. Issue d'un milieu économique aisé, l'enfant préfêre "le cricket, les barres, l'équitation et courir les champs"; elle raffole de "tous les jeux de garçons "qu'elle privilégie au détriment "des poupées"14. Aussi l'héroïne de Jane Austen, dont l'enfance contrarie les sphères genrées traditionnelles, doit-elle suivre une session intensive de

12 Jane Austen, Catberine Morland. Nortbanger Abbey, op. cit., p. 9.

13 Annie Goldmann dresse par exemple le même bilan quand elle analyse la littérature du $\mathrm{xIX}^{\mathrm{c}}$ siècle et ses personnages féminins pris au piège de leur formation idéologique: $\cdot \mathrm{Au} \mathrm{XXX}^{e}$ siècle, nous l'avons vu, elles sont toutes - à part Gervaise - en dehors de la vie économique. Elles se situent donc au niveau de la valeur d'usage, c'est-à-dire au niveau de la sensibilité, de l'affectivité. Annie Goldman, Rêves d'amour perdus. Les femmes dans le roman du $19^{\circ}$ siècle, Paris, Denoël/Gonthier, 1984, p. 193.

Jane Austen, op. cit., p. 10-11. 
féminité: "[...] de quinze à dix-sept ans, elle suivit un régime d'héroïne $[\ldots]^{15}$. La force satirique dévoile ici la construction sociale de la féminité et la stratégie textuelle vise explicitement la prise de conscience de ce processus. Autrement dit, le texte ne plonge pas son lectorat dans une féminité donnée mais il insiste bien au contraire sur le fait qu'elle est acquise par des pratiques sociales très concrètes. Suivre un régime d'hérö̈ne signifie - et ici nous sommes dans le régime politique de la féminisation apprendre par cour "[...] qu'une jeune femme qui aime est toujours semblable... à la Résignation sur un piédestalsouriant à la douleur ${ }^{16}$. Il s'agit là évidemment d'une satire du roman sentimental si caractéristique du travail littéraire de Jane Austen mais qui en révèle à juste titre l'implicite idéologique. Alors que l'idée moderne d'amour remplit la fonction de faire croire à une innéité hétérosexuelle, ce récit du $\mathrm{XIX}^{\mathrm{e}}$ siècle nous en montre la formation sociale. Et la narration ironise superbement quand, une fois la session terminée, elle doit actualiser le terminus a quo de la féminité : "Sur sa route, le destin doit susciter et suscitera un héros" ${ }^{17}$. La rencontre avec le héros se réalise au sein d'une société dont le principe matériel et le dessein fortement pensé servent l'administration sentimentale. Chez Jane Austen, rien ne relève du hasard, de la rencontre fortuite, tout dément la supposée naturalité du sentiment amoureux. Les parents cherchent en effet à "caser" une fille qu'ils envoient dans un espace social plus propice à organiser son destin obligé. N'ayant aucun prétendant en vue dans son environnement immédiat, Catherine est envoyée à Bath où les bals et mondanités vont favoriser son insertion dans l'ordre du sentiment. Ainsi révélée la finalité matérielle du mariage, l'amour dans Catberine Morland. Northanger Abbey ne correspond guère à sa définition encyclopédique ${ }^{18}$ qui lui prête une nature désintéressée.

Le projet romanesque vise explicitement la construction raisonnable de la relation sentimentale, et plus précisément de l'intérêt fondamental à hypercodifier l'identité féminine. Les

15 Ibid., p. 12.

16 Ibid.

17 Ibid., p. 13.

18 Le scénario encyclopédique est une structure qui se fonde sur des données socialement acceptées en raison de leur constance statistique et qui sert en fait à représenter une situation stéréotypée. Voir Umberto Eco, Lector in fabula, traduit de l'italien par Myriem Bouzaher, Paris, Grasset, 1985, p. 87-109. 
conséquences en sont bien sûr oppressives et l'humour qui les souligne ici se fait grinçant: "Et maintenant, je puis envoyer Catherine vers la couche d'insomnie qui sied à une héroïne de roman. Qu'elle se tienne pour satisfaite si, au cours des trois mois qui vont suivre, elle a une nuit de sommeil calme. ${ }^{19}$. Si la tradition sentimentale a pour visée de cacher le caractère coercitif de ce principe identitaire, la voix acerbe de Jane Austen fait entendre un autre son de cloche. L'amour y perd ses effets d'idéalisation, la féminité son auréole éthérée. Le "bonheur parfait. qui termine le roman nous laisse un goût de fiel, celui-là même qui opère quand un discours sait montrer les causes profondes de la différenciation sexuée, à savoir enfermer le personnage féminin dans une trajectoire normative: "Dans le mariage, l'homme est supposé subvenir aux besoins de la femme, la femme rendre la maison agréable à son mari. Il ravitaille et elle sourit "20. Ainsi parle le héros que Catherine aimera pour la vie. Le récit des amours étant terminé, Jane Austen laissera à son lectorat la possibilité de juger par lui-même la morale de son histoire: "Je laisse à qui peut s'intéresser à ce genre de spéculations le soin de déterminer si ce livre prône la,tyrannie paternelle ou la désobéissance filiale" 21 .

L'humour est aussi corrosif dans le roman québécois que Louise Leblanc écrit en 1983. Les deux siècles qui séparent Jane Austen et Louise Leblanc n'ont pas, malheüreusement, libéré les femmes de l'idée moderne d'amour. Depuis les années 1950, la paralittérature sentimentale constitue une véritable multinationale qui gère autant leur cour que leur conscience. Le roman de Louise Leblanc, $371 / 2 A A$, se veut une parodie du corpus Harlequin. Il ne fait aucun doute que les effets du marketing Harlequin produisent un corpus qui témoigne d'une continuité avec l'idée moderne d'amour, même si, nous l'avons souligné en introduction, il n'en détient pas l'exclusivité. Cependant, la paralittérature procède par une reproduction de lieux communs: ses personnages sont canoniques, les scénarios et motifs qu'il met en scène répétitifs 22 . Dans ce sens, le roman Harlequin reprend le

19 Ibid., p. 92.

20 Ibid., p. 79.

21 Ibid., p. 253.

22 Nous renvoyons pour une analyse sémiotique du corpus Harlequin à l'ouvrage collectif suivant: La corrida de l'amour. Le roman Harlequin, sous la direction de Julia Bettinotti, C.D.E.L., Montréal, UQAM, 1986, 160 p. 
scénario encyclopédique de l'homme et de la femme dont il organise en cent cinquante pages la destinée irrépressible. Parant au plus pressé, le déroulement sériel des motifs donne lieu à une structure narrative fixe dont il convient de souligner la cohérence: Harlequin pense et prévoit ce qui du hasard ou d'une imagination scripturale ferait chavirer son parcours obligé. Aussi y retrouve-t-on cinq séquences narratives dont la combinaison invariable se lit dans un déroulement intratextuel fixe.

Comme toute parodie qui se respecte, le roman de Louise Leblanc emprunte cette formule narrative en se distanciant de l'objet de référence par l'exagération et la caricature. Le scénario de base de la rencontre entre le héros et l'héroine, la confrontation polémique qui s'ensuit ainsi que l'émergence du sentiment amoureux et le mariage final représentent donc les macropropositions de cet univers romanesque. Comme chez Jane Austen, la formation de la relation amoureuse fonde l'enjeu principal du récit qui focalise cependant sur les deux protagonistes. Loïc De La Manne, richissime industriel, poursuit l'héroïne Fleur-Ange par force de violence qu'elle reçoit sans coup férir. Il la frappe sauvagement, il la torture mais il lui sauve la vie quand elle risque de la perdre. Loïc De La Manne représente en fait le héros dur, froid et cynique du roman Harlequin mais dont l'enfance révèle une blessure émotive enfouie au plus profond de son être viril. Le récit s'attaque ici aux explications "psy" qui justifient les rapports de pouvoir. Un drame a obscurci les années d'enfance de Loïc, drame bien sûr directement relié à une mauvaise mère, voilà qui rationalise les causes du pouvoir qu'il exerce contre les femmes. Le récit insiste longuement sur cette justification:

- Pervenche, la mère de Loïc, était très belle, mais c'était une fermme capricieuse, fantasque, changeante, vaniteuse, futile, frivole, inconséquente, irresponsable $[\ldots]$.

Dix minutes plus tard...

-... égoïste, superficielle, calculatrice, [...].

•[...] son père, qui était un être bon, sensible, doux, généreux, indulgent, attentionné, [...].

Vingt minutes plus tard...

.... simple, juste, brave, courageux, tolérant [...]. . 23 
16

La fonction critique du comique exprimé ici est hautement efficace et va bien au-delà du corpus parodié. Ainsi mise à découvert, nous retrouvons la marque déposée d'un discours social dominant qui occulte, toujours en les justifiant, les rapports de pouvoir hommes/femmes. Â ce niveau, la stratégie adoptée par Louise Leblanc traque parfaitement la doxa qui travaille les représentations sexuées. Si le portrait du héros intègre l'aire psychologisante, celui de l'héroïne s'érige (toujours sur un mode humoristique) en fonction des critères naturalistes. À l'instar du parti pris textuel de Jane Austen, Louise Leblanc privilégie en effet une héroïne qui doit, sous les directives de son oncle, se comporter en "garçon" pour échapper aux commérages du village. Cependant, si le parti pris est le même que dans le roman de Jane Austen, il n'a pas la même fonction. Louise Leblanc critique ici l'idéologie naturaliste ${ }^{24}$ en exacerbant les traits somatiques de son personnage. Toutes les parties du corps de FleurAnge sont ainsi troublées par une féminité larvée mais incontrôlable. Sa nature féminine se cadence au son de *battements accélérés du coeur", "d'élans spontanés", de "bouillonnements intempestifs", bref elle ne peut échapper à ce corps qui parle. Inscrite dans ses gènes, sa féminité bouillonne en elle comme une lave incandescente qui n'aura d'apaisement que dans la relation avec le héros. Étant née pour être avec son héros, le personnage de Fleur-Ange cristallise la grammaire ritualisée des héroïnes de romans dont la seule signification se restreint au domaine de la vie privée. Rares en effet sont les récits qui élargissent les sphères d'action imparties aux personnages féminins. Dans $371 / 2 A A$, il est impossible de passer à côté de ce rapport critique tant l'aspect parodique élucide à outrance cette prescription sociosexuée. Ia fin du récit reviendra sur ces traits isotopes qui constituent le système de places genrées des personnages. Le roman propose une finale idyllique actualisée par le mariage de Loïc et Fleur-Ange. Et tout se passe ici comme si les rapports de pouvoir au sein desquels émerge le sentiment amoureux étaient oubliés. Le roman installerait quasiment le public-lecteur à la

24 Nous renvoyons aux travaux de Colette Guillaumin pour l'analyse de l'idée de Nature: -Ce seraient les •capacités - internes (donc naturelles) qui détermineraient les faits sociaux, ce qui revient à l'idée de déterminisme endogène dans les rapports humains [...]•. Colette Guillaumin, - Race et nature: système des marques. Idée de groupe naturel et rapports sociaux •, Pluriel, $\mathrm{n}^{\circ} 11,1977$, p. 48. 
même place idéologique que le roman Harlequin, n'était-ce une digression qui vient, interrompre le fil idéalisé de l'union sacrée: "Comme un soleil qui éclate, la passion pulvérisa son corps, appelant l'éternelle capitulation de la femme devant l'homme " 25 . En décochant cette ultime flèche, le récit évacue la possibilité de passer outre la fonction répressive de l'amour moderne tout en soulignant l'impossibilité de le penser en dehors de cette féminité qui lui est si nécessaire pour assurer la dite fonction.

Louise Leblanc comme Jane Austen sont deux romancières dont les productions opèrent, l'une dans la parodie, l'autre dans la satire, une transgression de l'idée moderne d'amour. En démontant le piège essentialiste qui alloue à l'amour un caractère naturel, leur récit montre les politiques spécifiques de son élaboration. Avec Elfriede Jelinek, nous nous retrouvons dans un sarcasme cru et froid; les héroïnes de son roman intitulé les amantes sont prisonnières d'un univers sentimental... concentrationnaire.

C'est aussi avec l'intention polémique de s'attaquer à l'amour, celui des romans-photos, qu'Elfriede Jelinek manie sa plume romanesque : «si quelqu'un a un destin, alors c'est un homme. si quelqu'un se voit imposer un destin, alors c'est une femme. 26 . Sans aucune lueur d'espoir, le roman raconte l'histoire de paula et brigitte, deux ouvrières qui travaillent dans une usine de textile. Pour échapper à l'univers gris de l'usine, une seule possibilité s'offre à elles: l'univers tout aussi gris du mariage. Dans l'un ou l'autre cas, le présent et l'avenir sont bien loin de ressembler à une idylle:

à l'avenir paula et erich seront parfois complémentaires; par exemple lorsqu'erich donnera des coups et que paula recevra des coups, ou lorsqu'erich sera malade et que paula le soignera, ou lorsqu'ils iront ensemble scier du bois, ou lorsque paula fera la cuisine et qu'erich mangera. ${ }^{27}$

brigitte pense qu'il lui arrivera bien de se rebiffer, mais, qu'en gros, heinz a raison et qu'elle fera ce qu'il dit. ${ }^{28}$

25 Ibid., p. 198.

26 Elfriede Jelinek, Les amantes, op. cit., p. 7. Nous respectons ici le code scriptural de ce texte, notamment l'absence de majuscules.

27 Ibid., p. 84.

28 Ibid., p. 192. 
18

Le roman se déroule à l'instar de ces citations et il est facile d'imaginer, pour qui ne l'aurait pas lu, le ton ironique qui le nourrit. En fait, rien n'échappe à l'œil froid de la narration omnisciente. Toute illusion sentimentale en est absente ou plutôt détruite par une réalité dure et cynique, à la limite d'un ordre insupportable des choses. Procès-verbal de "l'esclavagisme moderne" "29, le discours romanesque dévoile ce qui doit resté caché, ce qui est encore tabou social. Dans Les amantes, l'amour représente en effet un univers carcéral qui fixe les personnages féminins à une place immuable: le mariage, les tâches ménagères, la maternité. Susi, seule intellectuelle, intégrera elle aussi le clan féminin même si cette intégration se fait avec plus de "distinction":

susi par contre veut rester mince grâce à la gymnastique, afin d'être et de rester pour son mari une amante agréable. [...] susi veut aussi être une partenaire sur le plan intellectuel. c'est bon aussi pour les enfants quand la mère est à la hauteur, intellectuellement. ${ }^{30}$

Pour assurer son efficace idéologique, l'idée moderne d'amour doit s'appuyer sur un principe doxal qui ne remette pas en question l'identité sexuelle. Ici, au contraire, Jelinek insiste sur la socialité qui préside à la construction des catégories de sexe. Chacune s'inscrit dans un système qui l'infléchit de façon déterminante. Le procès de signification instruit des portraits sémantiques différenciés et dont la répartition binaire est structurée par l'amour. Le roman de Jelinek met ainsi à découvert la façon dont s'organise structurellement la thématique sentimentale dans sa version dominante. Certes, il s'ensuit un texte provocant, un antiroman d'amour en quelque sorte qui s'attaque aux représentations traditionnelles. Dans ce sens, l'amour n'est plus un îlot romantique mais un outil de répression extrêmement violent auquel il n'est plus possible de rêver. En lui faisant perdre ses effets d'idéalisation, la trajectoire romanesque le place dans la matérialité de l'oppression des femmes: «que l'amour ne soit lié qu'au travail, personne n'aime à l'admettre" ${ }^{31}$. Des trois produc-

29 L'expression est de Jelinek : - Lust est une forte critique sociale, un exemple démontrant les mécanismes de l'esclavagisme moderne. Si la remarque s'applique à un autre de ses romans, elle convient tout aussi bien aux Amantes. Elfriede Jelinek. Sur la brèche, sans fard avec artifices *, Nuit blanche, n ${ }^{\circ}$ 54, décembre 1993, janvier/février 1994, p. 36.

30 Elfriede Jelinek, Les amantes, op. cit., p. 209-210.

31 Ibid, p. 38. 
tions romanesques, celle d'Elfriede Jelinek reste la plus dérangeante et peut-être celle qui n'alloue aucun droit d'asile à l'amour. Sa principale force de désintoxication tient non seulement à l'ironie qui s'y déploie mais aussi à une construction antithétique du Bildungsroman. En effet, l'apprentissage de l'amour par les héroïnes, au lieu de les amener vers une connaissance d'elles-mêmes et une découverte du monde, les emprisonne dans un univers de plus en plus rétréci et étriqué.

Chaque parcelle de cet univers est passé à la loupe et aucune des sphères conventionnellement féminines ne propose une échappée. Il s'agit là d'une subversion destructurant l'idéalisme des discours qui masquent les techniques coercitives sur lesquelles repose l'exercice de l'amour moderne. Il y a une certaine horreur à dévoiler radicalement ce qui se cache d'abjections derrière cette marque déposée dans le genre humain: un rapport d'oppression organisé, c'est là l'objectif fondamental de ce roman d'abominer l'amour. À ce niveau, Elfriede Jelinek remet en question une féminité sacro-sainte en socialisant ses héroïnes à l'intérieur d'une quotidienneté éprouvante. Telles des bêtes de somme, elles fournissent un travail qui ne peut plus faire illusion parce que la vision du monde qui s'en dégage s'élabore à même une réalité qui opprime les héroïnes. Le récit se terminera sur un épilogue dont l'ironie dément le propos énoncé: "connaissez-vous ce BEAU pays avec ses monts et ses vaux? au loin de belles montagnes le délimitent. il a un horizon, peu de pays peuvent en dire autant ${ }^{3}$.

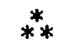

Elfriede Jelinek a déjà été agressée dans son pays pour avoir osé écrire de tels romans et il ne faut pas s'en étonner. On se souvient de notre citation inaugurale et des résonances politiques qu'elle implique. La sémiosis sentimentale fait croire à la naturalité des rapports entre les sexes, sans jamais montrer que les hommes et les femmes sont des construits déterminés par un rapport social, en l'occurrence de pouvoir. Les trois romans que nous avons parcourus dans le cadre restreint de cet article en dévoilent, quant à eux, le procès de signification. S'il va sans dire 
que l'amour est au coeur de l'organisation sociale moderne - et la multitude des discours qui le thématisent ou le théorisent révèle en quelque sorte cette importance - le matériau textuel qui nous est donné à voir dans la tradition sentimentale joue sur des mécanismes qui tabouisent aussi bien la nature rationnelle de l'objet que sa fonction politique. Dans sa version moderne, la culture occidentale a constamment tenté de rendre invisibles les traces de cette histoire que l'on tient fermement à idéaliser alors qu'elle est en réalité extrêmement répressive.

De nombreux aphorismes gravitent autour de l'amour mais il en est un qui esquisse le creuset épistémique dans lequel théorie et fiction puisent leurs nébuleuses déréalisations : "En amour, cécité est souvent fille de nécessité». Aussi la société en parle-t-elle par nécessité de cécité en quelque sorte, pour reproduire ad nauseam la censure sur ce qui ne doit pas se dire, sur ce qui doit demeurer dans l'ordre d'un impensable sociétal. Jane Austen, Louise Leblanc et Elfriede Jelinek rompent ce contrat implicite en traquant dans leurs productions le donné ontologique. De façon distincte, leur projet romanesque cerne au plus proche les démons intérieurs de l'amour moderne, il en révèle le machiavélique dessein et leur geste dans ce sens milite en sa défaveur. Elles font œuvre de transgression en touchant les bases structurelles et l'ancrage matériel de l'objet social. Toutes les trois ont compris l'importance de remettre en question le principe essentialiste qui alloue à la féminité un caractère naturel. Si le texte de Jane Austen focalise sur la formation intensive de la construction sociale, celui de Louise Leblanc en raille l'étiquette naturaliste; quant à Elfriede Jelinek, son roman présente l'insoutenable vision de l'univers carcéral du sentiment pour les personnages féminins. La sémiosis à l'œuvre remplit alors une fonction critique en osant confronter le "déjà-là " d'un monde figé par une praxis qui a imposé le cadre normatif des schèmes sexués et l'obligation de leuŕ rencontre. C'est bien le caractère dogmatique du sens amoureux qu'elles mettent sur la sellette et ce sont ici les croyances implicites et le champ des évidences qui s'en trouvent chamboulés. En mettant au jour les règles et les marques qui organisent la féminité amoureuse, les trois romans font front commun avec l'énoncé féministe de Firestone. Et en osant dire le non-dit, ces romancières nous permettent d'échapper un peu à la tradition sentimentale moderne, qui, même en cette fin de $x x^{e}$ siècle, est encore si prégnante. L'enjeu était de taille mais l'interstice de résistance qu'elles nous offrent vaut son pesant d'or. 